\section{Positive Deviance and Hand Hygiene: More Questions than Answers}

To the Editor-Optimizing adherence to hand hygiene recommendations has been problematic in many healthcare settings. We read with interest the article by Marra et $\mathrm{al}^{1}$ relating their experience with a positive deviance (PD) approach to improving hand hygiene adherence. The authors should be commended for adding to our understanding of hand hygiene and the PD strategy for improving adherence, but a number of limitations should be noted and several questions should be addressed.

Because actual opportunities for hand hygiene were not monitored (use of alcohol-based gel was measured), the level of hand hygiene adherence in the study is not known. In the east step-down unit (SDU), the rate of alcohol-based gel use increased from 47 dispensations per patient-day to approximately 60 dispensations per patient-day, whereas in the west SDU, the rate of gel use initially decreased (before PD was introduced) and then returned to baseline (with PD). In our own study in a critical care unit, we noted opportunities for hand hygiene at a rate of 295 opportunities per patient-day, ${ }^{2}$ and Pittet et $\mathrm{al}^{3}$ noted a rate more than twice as high as ours. In unpublished observations in our SDU, we noted opportunities for hand hygiene at a rate of 276 opportunities per patient-day. Thus, the rate of gel use observed by Marra et $\mathrm{al}^{\mathrm{l}}$ at its highest (62 dispensations per patient-day) may have been woefully inadequate; an explanation by the authors for the possible reasons for such a low rate would be welcome.

It is not clear whether the PD approach worked. It would be of interest to know the change (mean difference and standard deviation) in the number of alcohol-based gel aliquots dispensed between the first phase and the second phase in the west and the east SDUs separately; these 2 analyses would provide the "over-time baseline changes" (use of gel without PD) and the "over-time intervention changes" (use of gel with $\mathrm{PD}$ ), respectively. Then, the over-time baseline changes in the west SDU should be statistically compared with the over-time intervention changes in the east SDU to better determine whether the effect of PD was confounded by the expected (natural course) over-time baseline changes in the use of gel. Also, a comparison analyzing the changes from the first phase (baseline) to the third phase (PD in both units) should produce significant changes (increase in gel use) within each unit but no significant changes between units, because both underwent intervention with PD in the third phase.

It is not clear what types of healthcare-associated infections were reduced. The authors showed quite nicely that various device-associated infections were not significantly impacted, but they did not relate what types of infections were reduced. Also, similar to our suggestion above, it would be important to have a statistical comparison of the device-associated-infection and the healthcare-associated-infection incidence density changes in the second and third phases, separately, compared with the first phase (baseline infection rate). We should expect significant infection incidence density changes in the east SDU in the second phase and in both SDUs in the third phase, but no difference between units when comparing first and third phases.

It is not clear whether PD results in a sustained effect. An observation period of only 3-6 months is simply not long enough to judge. As the authors note, the generalizability of this approach is unknown.

An extensive list of potential confounders should be noted. The overall antibiotic use (number of defined daily doses per 1,000 patient-days) in the first unit to undergo PD intervention increased dramatically (by $52 \%$ ), even though the number of healthcare-associated infections decreased by almost $31 \%$. The authors did not further characterize this counterintuitive observation. If anything, one would expect antibiotic use to decrease in response to successful prevention of healthcare-associated infection. The length of stay decreased dramatically in both units (by more than $20 \%$ ) during the first intervention phase. No explanation for this observation was offered, and it might indicate other forces at work. Severity of illness is an obvious confounder that may have been indirectly measured by the nurse activity score. This score differed significantly between units in 2 of the 3 observation periods, decreased steadily by $11 \%$ in one unit over the course of the study, and fluctuated in the other unit.

Thus, as is often the case with even the best studies, we are left with many questions unanswered. A great deal of work remains to be done to determine how best to measure hand hygiene rates, to improve adherence to hand hygiene recommendations, and to ascertain what impact hand hygiene has on healthcare-associated infection.

\section{ACKNOWLEDGMENTS}

Potential conflicts of interest. Both authors report no conflicts of interest relevant to this article.

Mark E. Rupp, MD; Andre C. Kalil, MD

From the Department of Internal Medicine, University of Nebraska Medical Center, Omaha (both authors).

Address reprint requests to Mark E. Rupp, MD, 984031 Nebraska Medical Center, Omaha, NE 68198-4031 (merupp@unmc.edu).

Infect Control Hosp Epidemiol 2010; 31(9):978-979

(C) 2010 by The Society for Healthcare Epidemiology of America. All rights reserved. 0899-823X/2010/3109-0020\$15.00. DOI: $10.1086 / 656204$

\section{REFERENCES}

1. Marra AR, Guastelli LR, Pereira de Araujo CM, et al. Positive deviance: a new strategy for improving hand hygiene compliance. Infect Control Hosp Epidemiol 2010;31:12-20. 
2. Rupp ME, Fitzgerald T, Puumala S, et al. Prospective, controlled, crossover trial of alcohol-based hand gel in critical care units. Infect Control Hosp Epidemiol 2008;29:8-15.

3. Pittet D, Mourouga P, Perneger TV. Compliance with handwashing in a teaching hospital. Ann Intern Med 1999;130:126-130.

\section{Reply to Rupp and Kalil}

We appreciate the comments by Rupp and Kalil ${ }^{1}$ with regard to our article that analyzed positive deviance (PD) strategy for improving hand hygiene adherence. ${ }^{2}$ We will clarify some aspects discussed by Rupp and Kalil. ${ }^{1}$

First, the most notable of the special features is the study design. There is a need for improved study design in reports of hand hygiene interventions. A Cochrane review in 2007 dismissed nearly all the literature on hand hygiene interventions because of inadequate study design. ${ }^{3}$ We believe that our manuscript would not be dismissed by the Cochrane reviewers. Our study was an experimental study-that is, a controlled trial developed in 2 adult 20 -bed step-down units (SDUs) with the same physical layout-east (intervention) and west (control) wards of the same hospital at the same time. Comparisons must be done between SDUs during the study periods (phase 1, east SDU vs west SDU; phase 2, east SDU vs west SDU; and phase 3, east SDU vs west SDU). That is the reason for not applying the "over-time baseline changes" and the "over-time intervention changes," as suggested by Rupp and Kalil. ' We also incorporated PD for improving hand hygiene in our hospital in 2009; at that time, the over-time baseline changes were shown to our staff (the positive deviants) during their meetings. After consideration of the comments about healthcare-associated infections (HAIs) by Rupp and Kalil, ${ }^{1}$ the comparison must be done between SDUs during the study periods.

In our prospective, controlled trial, we monitored HAIs, not only device-associated HAIs (eg, bloodstream infection associated with a central venous catheter) but also non-device-associated HAIs (eg, surgical site infection). The PD approach worked, resulting in a significant improvement in rates of hand hygiene adherence, which was associated with a decrease in the incidence of HAIs (both those associated with a device and those not associated with a device).

Second, the infection rate in the SDU is more similar to the infection rate in the ward setting than to the infection rate in an intensive care unit. ${ }^{4}$ Thus, it is difficult to compare the number of opportunities for hand hygiene in our study with the number of opportunities in studies performed in intensive care units. The studies were totally different. We monitored the use of only alcohol-based hand gel with the electronic hand-wash counter, but we had the consumption data for both chlorhexidine and alcohol-based hand gel. Direct observation could change the behavior of healthcare workers when they know that they are being observed. The Hawthorne effect would confound an observational study in which handwashing is being documented. Rupp and Kalil ${ }^{1}$ may have misunderstood the concept of opportunities for hand hygiene, particularly when they mention a rate of 295 opportunities per patient-day measured by observers for hand hygiene (we did not find these published results in Rupp et $\mathrm{al}^{5}$ ) and compare it with our highest rate of use of gel measured by electronic hand-wash counters ( 62 dispensations per patient-day). In our entire 9-month study, we had a great number of episodes of hand hygiene by the electronic handwash counters $(506,111$ episodes of hand hygiene!), whereas Rupp et $\mathrm{al}^{5}$ have only 3,678 opportunities for hand hygiene. This comparison is showing different scenarios. Again, these are totally different. This comparison does not make any sense.

Third, we agree that there is a paucity of evidence about sustained improvements in rates of hand hygiene adherence. We do not believe that an observation of improvement during 6 months is sufficient to be considered sustained. However, we believe that, as a first observation, it warrants further investigation to establish whether PD could yield sustained improvement in rates of adherence to hand hygiene. In fact, we continue to use the PD program in both SDUs (west and east), with approximately 40,000 gel aliquots dispensed per month in each SDU. In December 2009, no bloodstream infections, no urinary tract infections, and no tracheostomyassociated cases of pneumonia were reported in the SDUs.

Fourth, in the second phase the 2 SDUs had no distinction between patients, according to the Nursing Activities Score ${ }^{6}$ : 46.7 in the east (intervention) SDU versus 46.3 in the west (control) SDU. The difference in antibiotic consumption does not reflect a difference between the patient populations. The mean Nursing Activities Scores for these 2 populations are very similar. In the third phase, the antibiotic consumption in the east SDU was higher than that of the west SDU; however, PD was implemented in both SDUs, the Nursing Activities Score was significantly higher in the east SDU (51.1 vs 43.7 ), and no difference between the infection rates was detected (7.3 vs 5.4 infections per 1,000 patient-days).

We must accept, though, that the study raises a number of new and intriguing questions. However, in spite of the fact that there is much more to learn about hand hygiene, our study seems to answer some questions and to offer new solutions to this critical, world-wide issue. Hence, we feel that the title of the letter by Rupp and Kalil ${ }^{1}$ does not fairly account for the contributions of our article.

\section{ACKNOWLEDGMENTS}

Potential conflicts of interest. All authors report no conflicts of interest relevant to this article.

Alexandre R. Marra, MD; Miguel Cendoroglo Neto, MD; Oscar Fernando Pavão dos Santos, MD 